\title{
Optical fine-needle biopsy approach for intraoperative multimodal diagnostics in minimally invasive abdominal surgery
}

Ksenia Kandurova, Elena Potapova, Valery Shupletsov, Igor Kozlov, Evgeniya Seryogina, et al.

Ksenia Kandurova, Elena Potapova, Valery Shupletsov, Igor Kozlov, Evgeniya Seryogina, Viktor Dremin, Evgeny Zherebtsov, Alexander Alekseyev M.D., Andrian Mamoshin M.D., Andrey Dunaev, "Optical fine-needle biopsy approach for intraoperative multimodal diagnostics in minimally invasive abdominal surgery," Proc. SPIE 11079, Medical Laser Applications and LaserTissue Interactions IX, 110791C (22 July 2019); doi: 10.1117/12.2526747 


\title{
Optical Fine-Needle Biopsy Approach for Intraoperative Multimodal Diagnostics in Minimally Invasive Abdominal Surgery
}

\author{
Ksenia Kandurova*a, Elena Potapova ${ }^{\mathrm{a}}$, Valery Shupletsov ${ }^{\mathrm{a}}$, Igor Kozlov ${ }^{\mathrm{a}}$, Evgeniya Seryogina ${ }^{\mathrm{a}}$, \\ Viktor Dremin ${ }^{\mathrm{a}, \mathrm{b}}$, Evgeny Zherebtsov ${ }^{\mathrm{a}, \mathrm{b}}$, Alexander Alekseyev ${ }^{\mathrm{c}}$, Andrian Mamoshin ${ }^{\mathrm{a}, \mathrm{d}}$, \\ Andrey Dunaev ${ }^{\mathrm{a}}$ \\ ${ }^{a}$ Research and Development Center of Biomedical Photonics, Orel State University, Orel, Russia; \\ boptoelectronics and Measurement Techniques Unit, University of Oulu, Oulu, Finland; \\ 'Department of Anatomy, Operative Surgery and Emergency Medicine, Medical Institute, \\ Orel State University, Orel, Russia \\ ${ }^{\mathrm{d} O r e l}$ Regional Clinical Hospital, Orel, Russia
}

\begin{abstract}
The paper describes the methodology and technical implementation of a multimodal approach for optical diagnostics in hepatopancreatobiliary organs focal and diffuse neoplasms. Fine needle aspiration biopsy technique and following cytological examination show its effectiveness and safety but its performing takes several days. However, the problem of real-time analysis of pathological changes in tissues remains relevant. The solution suggested is implementing of optical biopsy methods (namely fluorescence spectroscopy and diffuse reflectance spectroscopy) in the form of fiber-optic probe compatible with standard biopsy fine needles. The special device was designed for this purpose to conduct optical measurements and compare the results with ones obtained by conventional biopsy. The proposed methodology seems promising for developing new diagnostic criteria for clinical practice.
\end{abstract}

Keywords: optical biopsy, fluorescence spectroscopy, diffuse reflectance spectroscopy, fine-needle aspiration biopsy, tumor, minimally invasive surgery

\section{INTRODUCTION}

Currently, the search for new diagnostic criteria for morphological verification of focal and diffuse neoplasms is one of the urgent problems of biophotonics applications for surgery. In particular, this is important in diagnosing of hepatocellular carcinoma. This type of cancer remains one of the most common malignant tumors and the leading cause of cancer-related deaths worldwide ${ }^{1,2}$. Early and accurate diagnosis of this pathology is necessary for the timely determination of treatment tactics, which leads to improving the prognosis of patients survival.

Morphological verification of focal and diffuse neoplasms is the basis for further diagnostic search and selection of surgical treatment tactics in such patients. Currently, the "gold standard" of morphological preoperative diagnosis of tumors is considered to be fine needle puncture and aspiration biopsy (FNAB) ${ }^{3}$. FNAB is the method of diagnosis of focal and diffuse tumors without resorting to open surgery. It involves taking cells from several areas of interest using a fine needle with a conventional or cutting edge for studying the obtained material under a microscope. Puncture with a fine needle is safe, atraumatic and allows one to obtain material without significant disruption of the integrity of pathological focus and surrounding tissues. However, classical FNAB involves sample preparation followed by cytological examination, which does not allow the doctor to obtain the necessary information in real time. In this regard, the search for new methods to determine the nature of pathological changes in tissues online remains relevant.

One of the rapidly developing approaches combining the advantages of conventional biopsy and compensating its downsides is so-called "optical biopsy"4. This technique includes the application of various spectroscopic and imaging methods for acquiring the information about biological tissues in vivo in real time without obtaining invasive sample.

*kandkseniya@gmail.com; phone +7 910 2682946; http://www.bmecenter.ru/en 
These methods allow evaluating such parameters as metabolic processes rate, chromophores content, blood perfusion and oxygenation.

Currently, many researchers use the method of fluorescence spectroscopy (FS) for studies of tissue or cellular metabolism. The use of FS in oncology is based on differences in the intensity and spectral composition of the fluorescence of healthy and malignant tissues when excited by laser radiation in the ultraviolet (UV) or visible range of the spectrum ${ }^{5,6}$.

Another technique called diffuse reflectance spectroscopy (DRS) provides information about light absorption in biotissues. DRS is used on its own or in combination with FS. This method allows differentiating malignant and benign tumors as well, as neoplastic tissue undergo significant architectural changes at the cellular and intracellular levels ${ }^{7}$.

The combination of several complementary optical modalities can give more valuable information for diagnosis and treatment ${ }^{8}$. For instance, the fluorescence normalization by reflectance spectra using DRS data allows increasing the diagnostic accuracy of fluorescence spectra by reducing the errors brought in by the absorption of tissue chromatophores ${ }^{9}$.

However, the application of several optical non-invasive methods for minimally invasive surgery and particularly for FNAB is associated with technological and methodological challenges. The absence of a commercially available system for the simultaneous registration the fluorescence spectra and diffuse reflectance in one diagnostic volume during FNAB does not allow one to quickly and effectively diagnose focal and diffuse tumors in real time during the procedure.

Previous studies on applying optical methods during minimally invasive operations in abdominal organs have shown the prospects of multimodal approach, however limitations due to anatomical features were revealed ${ }^{10,11}$. Implementation of this technology in fine needle format will provide wider access to neoplasm itself and will allow for using the results of conventional biopsy as a more accurate reference at the initial stages of development.

Therefore, the aim of the work was to develop the methodology and technical implementation of combined fluorescence and diffuse reflectance measurements during FNAB procedure for multimodal diagnosing of hepatopancreatobiliary organs focal and diffuse neoplasms.

\section{MATERIALS AND METHODS}

The in-house developed fiber optic system implementing the methods of FS and DRS (Fig. $1 \mathrm{a}, \mathrm{b})$ was designed for conducting experimental studies.

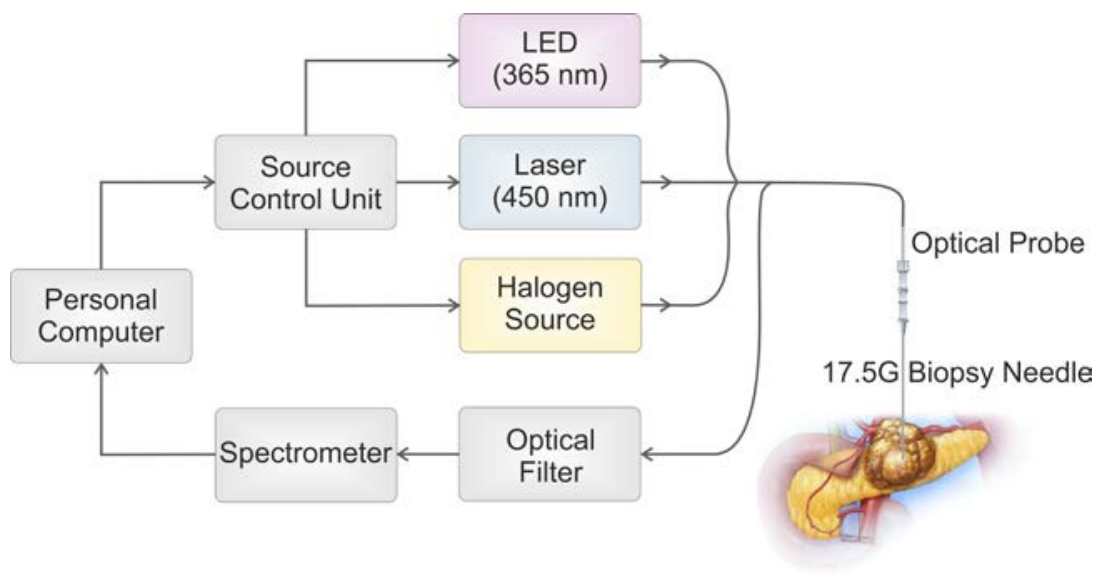

(a)

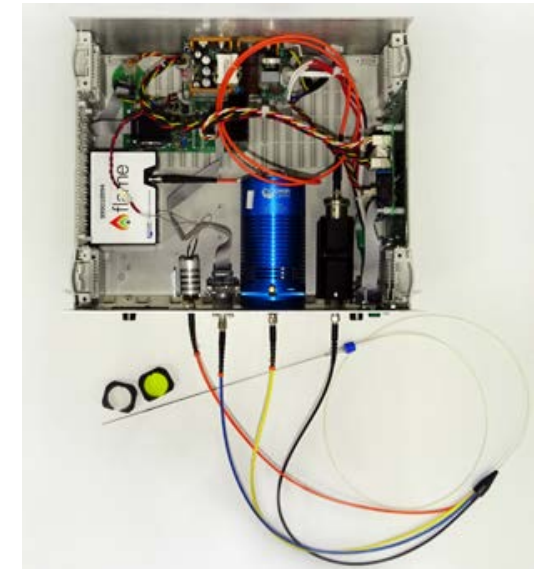

(b)

Figure 1. The schematic illustration (a) and photo (b) of the multimodal FS/DRS fine needle setup.

The FS channel includes two sources of monochromatic radiation: $365 \mathrm{~nm}$ LED and $450 \mathrm{~nm}$ laser diode. The choice of these wavelengths is caused by NADH and FAD coenzymes fluorescence excitation respectively. The accumulation of 
these substances in mitochondria during the tricarbonic acid cycle is related to oxidative phosphorylation. The content of NADH and FAD determines metabolism conditions and affects fluorescence spectra of tissues, therefore, fluorescence intensity parameter can be used to assess the intensity of metabolic processes ${ }^{6}$. The DRS channel includes HL-2000FHSA tungsten halogen light source (“Ocean Optics”, USA) with a wavelength range of 360-2400 nm.

The light collected from the tissue is filtered by the optical filter to attenuate backscattered radiation and then it is analyzed by a CCD FLAME spectrometer (“Ocean Optics", USA) in the range of 350-1000 nm. System control and further data processing are performed using a personal computer with custom developed software in Matlab program environment.

Both channels were combined in a single fiber optical probe sized of $1 \mathrm{~mm}$ outer diameter to be compatible with the 17.5G standard biopsy fine needle. The probe has 10 optical fibers. The nine transmitting ones $(100 \mu \mathrm{m}$ each) include three fibers connected to a halogen light source, three fibers connected to a laser diode and three fibers connected to an LED. The fibers are located around the central one $(200 \mu \mathrm{m})$, which delivers the collected light to spectrometer. The quantity and orientation of optical fibers inside the fiber-optic probe provide uniform and bright illumination of the diagnostic volume and allows reaching a high signal-to-noise ratio. The probe has a bevel angle of 20 degrees, which ensures reliable contact of the probe with dense tissues.

The proposed methodology assumes the application of designed setup during standard FNAB procedure under ultrasound control (Fig. 2). The surgeon introduces the fiber optical probe through 17.5G fine needle into the tumor. At the first stage, the operator turns on the necessary light source and put the corresponding optical filter. The optical radiation of monochromatic source induces fluorescence of various endogenous fluorophores. The radiation from the biological tissue is delivered by the transmitting fibers through the optical filter to the spectrometer. The visualization and further processing are performed on a personal computer. After the measurement, the operator can change the monochromatic source and filter or turn on the halogen lamp and remove the filter to record diffuse reflectance spectra. After that, the measurement cycle can be repeated a necessary number of times.

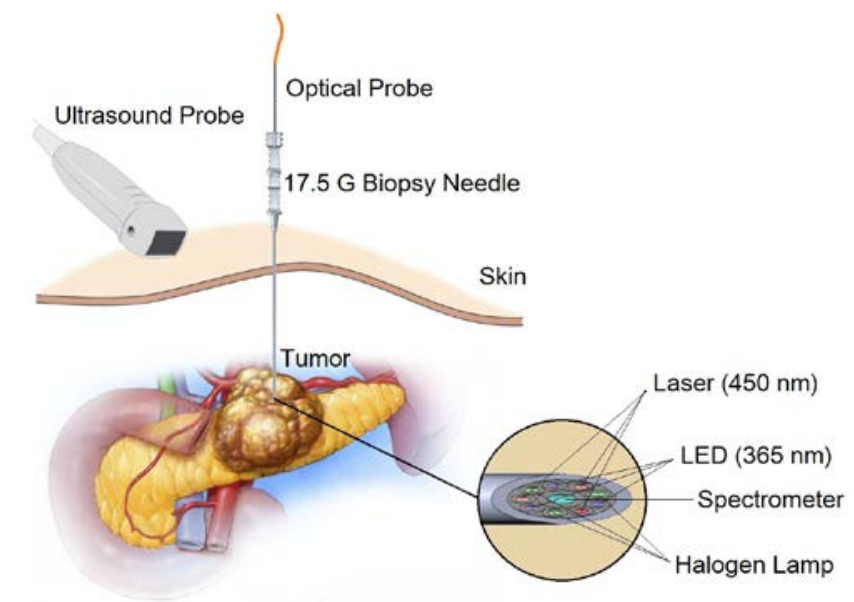

Figure 2. The scheme of measurement during standard FNAB procedure.

After optical measurements, the surgeon obtains the tissue sample from the same area for further cytological analysis. The comparison of two types of biopsies makes it possible not only to verify the presence of pathological changes in tissues but also to have a reference for more accurate interpretation of measurements results. The constantly extending database of fluorescence and diffuse reflectance spectra combined with the corresponding diagnosis by conventional biopsy will be used to develop new diagnostic criteria for clinical practice.

\section{RESULTS AND DISCUSSION}

Previously, a test study of the capabilities of the developed device on pathologically altered rat tissues was conducted ${ }^{12}$.

In this study, preliminary measurements using developed methodology and equipment were carried out at the department of interventional radiology of Orel Regional Clinical Hospital (Orel, Russia). The study was approved by the Ethics 
committee of Orel State University (record of the meeting №14 of 24.01.2019) and was carried out in accordance with the 2013 Declaration of Helsinki by the World Medical Association. The patients signed informed consent indicating their voluntary willingness to participate in the study. The measurements were performed during standard FNAB procedure in two patients (male, 45 y.o. and female, 74 y.o.) with supposed liver cancer. The studies were followed by the standard procedure of the biopsy sampling and histological examination (Fig. 3). The morphological picture corresponds to the small-cell undifferentiated carcinoma. The second sample contains fragments of liver tissue with areas of fibrinoid necrosis and complexes of low-grade adenocarcinoma.

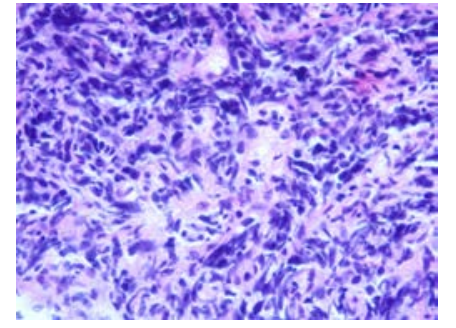

(a)

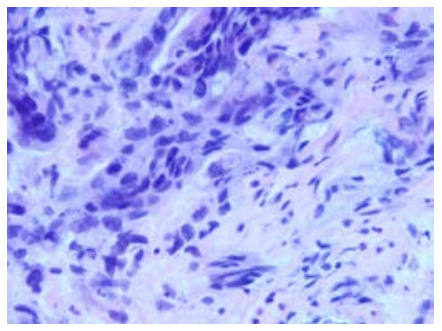

(b)

Figure 3. Histological examination of tumor tissues with haematoxylin and eosin staining at x40: patient 1 (a) and patient 2 (b).

The spectra were obtained three times from each area for further averaging (Fig. 4). The analyzed parameters were maximums of fluorescence intensity and diffuse reflectance intensities. Both cases showed similar differences in the registered spectra.
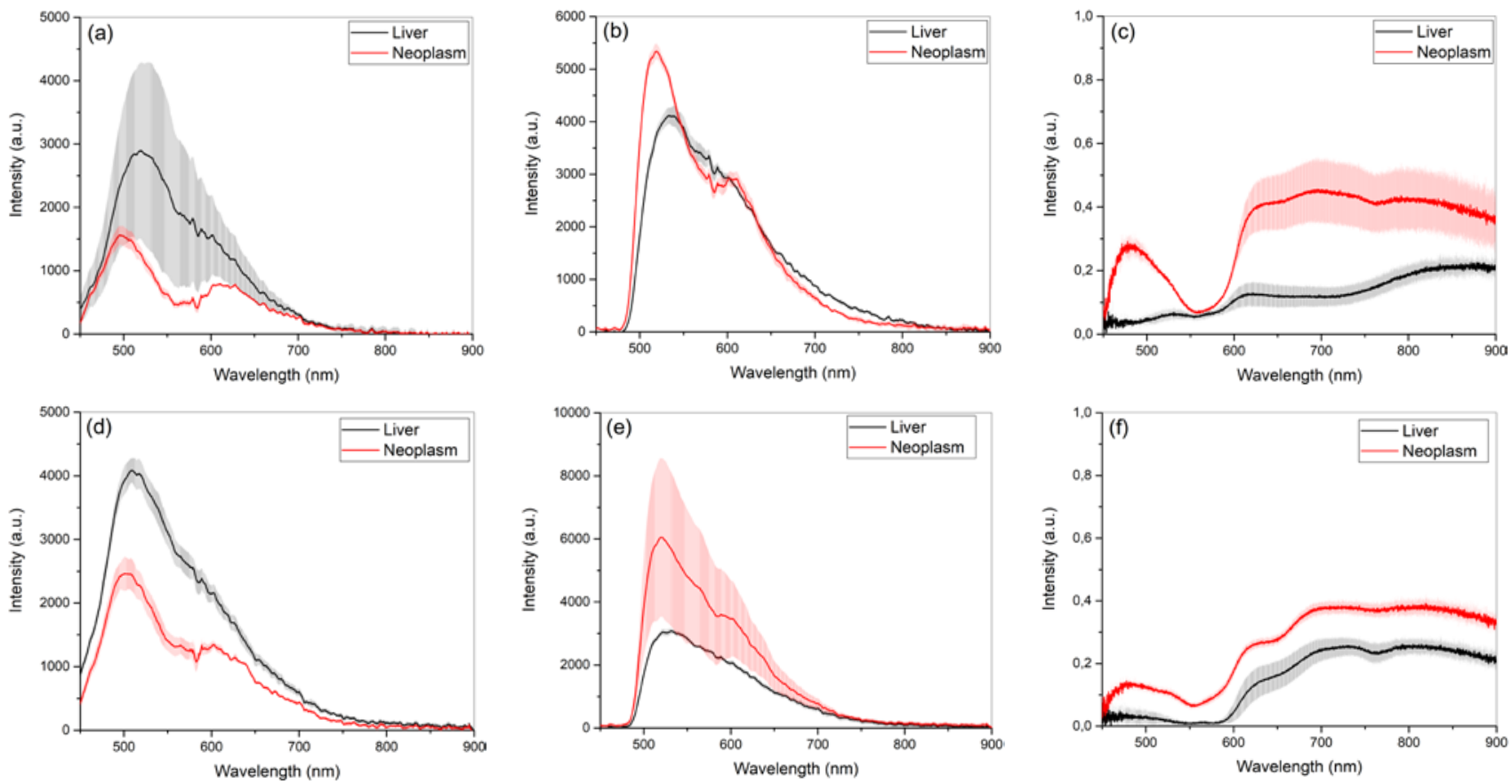

Figure 4. Fluorescence spectra at excitation wavelengths $365 \mathrm{~nm}, 450 \mathrm{~nm}$ and diffuse reflectance spectra, respectively, of normal (black line) and tumor tissue (red line) of patient 1 (a, b, c) and patient 2 (d, e, f).

The intensity of diffuse reflectance in tumors is higher than in parenchyma, which indicates the presence of morphological changes in the tissue. Low diffuse reflection in liver tissue may be associated with high blood content. The decrease of reflectance at $550 \mathrm{~nm}$ associated with deoxyhemoglobin and the absence of oxyhemoglobin absorption peaks at 540 and $580 \mathrm{~nm}$ indicates tumor tissues ischemia.

The fluorescence intensity at $365 \mathrm{~nm}$ is mostly higher in liver parenchyma and the fluorescence intensity at $450 \mathrm{~nm}$ is higher in tumor tissue. This may be caused by changes in the metabolic activity of cells in the tumor tissue, as well as a lower blood content that absorbs radiation, especially in the blue region of the spectrum. 


\section{CONCLUSION}

The application of multimodal approach including the methods of fluorescence spectroscopy and diffuse reflectance spectroscopy seems promising for the practice of guided surgery to objectify the criteria for selecting surgical tactics. Simultaneous registration of fluorescence and diffuse reflectance spectra carries the information about the corresponding parameters of metabolic processes and morphological structure of tissues, which is of interest for clinical practice. As the biopsy remains a valuable diagnostic method required to solidify the diagnosis, the necessity of real-time analysis determine the relevance of optical biopsy research. The proposed methodology of acquiring the optical parameters data and its correspondence with biopsy samples from almost the same diagnostic volume will provide the basis for the development of automatic classifier using the neural network. This will make diagnostics during minimally invasive surgical procedures faster and more accessible and will increase its accuracy and reliability.

\section{ACKNOWLEDGEMENTS}

This study was supported by the Russian Science Foundation under project № 18-15-00201.

\section{REFERENCES}

[1] Bosch, F. X., Ribes, J., Díaz, M. and Cléries, R., "Primary liver cancer: Worldwide incidence and trends," Gastroenterology 127(5), 5-16 (2004).

[2] Clark, T., Maximin, S., Meier, J., Pokharel, S. and Bhargava, P., "Hepatocellular Carcinoma: Review of Epidemiology, Screening, Imaging Diagnosis, Response Assessment, and Treatment," Curr. Probl. Diagn. Radiol. 44(6), 479-486 (2015).

[3] Gharib, H., Papini, E., Paschke, R., Duick, D. S., Valcavi, R., Hegedüs, L. and Vitti, P., “American Association of Clinical Endocrinologists, Associazione Medici Endocrinologi, and European Thyroid Association medical guidelines for clinical practice for the diagnosis and management of thyroid nodules,” J. Endocrinol. Invest. 33(5), 1-50 (2010).

[4] Kennedy, G. T., Okusanya, O. T., Keating, J. J., Heitjan, D. F., Deshpande, C., Litzky, L. A., Albelda, S. M., Drebin, J. A., Nie, S., Low, P. S. and Singhal, S., "The optical biopsy: A novel technique for rapid intraoperative diagnosis of primary pulmonary adenocarcinomas,” Ann. Surg. 262(4), 602-609 (2015).

[5] Rafailov, I., Palmer, S., Litvinova, K., Dremin, V., Dunaev, A. and Nabi, G., "A novel excitation-emission wavelength model to facilitate the diagnosis of urinary bladder diseases,” Proc. SPIE 9303, 93030W (2015).

[6] Tuchin, V. V., [Handbook of Optical Biomedical Diagnostics] SPIE Press, Bellingham, (2002).

[7] Vo-Dinh, T., [Biomedical photonics handbook: Biomedical diagnostics] CRC Press, Boca Raton, (2014).

[8] Dremin, V. V, Zherebtsov, E. A., Sidorov, V. V, Krupatkin, A. I., Makovik, I. N., Zherebtsova, A. I., Zharkikh, E. V, Potapova, E. V, Dunaev, A. V, Doronin, A. A., Bykov, A. V, Rafailov, I. E., Litvinova, K. S., Sokolovski, S. G. and Rafailov, E. U., "Multimodal optical measurement for study of lower limb tissue viability in patients with diabetes mellitus," J. Biomed. Opt. 22(8), (2017).

[9] Dremin, V. V., Zherebtsov, E. A., Rafailov, I. E., Vinokurov, A. Y., Novikova, I. N., Zherebtsova, A. I., Litvinova, K. S. and Dunaev, A. V., "The development of attenuation compensation models of fluorescence spectroscopy signals,” Proc. SPIE 9917, 99170Y (2016).

[10] Kandurova, K., Dremin, V., Zherebtsov, E., Potapova, E., Alyanov, A., Mamoshin, A., Ivanov, Y., Borsukov, A. and Dunaev, A., "Fiber-optic system for intraoperative study of abdominal organs during minimally invasive surgical interventions," Appl. Sci. 9(2), (2019).

[11] Kandurova, K., Dremin, V. V., Zherebtsov, E. A., Dunaev, A. V., Mamoshin, A. V., Alyanov, A. L., Muradyan, V. F. and Potapova, E. V., "Application of the fluorescence spectroscopy for the analysis of the state of abdominal cavity organs tissues in mini-invasive surgery,” Proc. SPIE 10685,106854P (2018).

[12] Dremin, V. V., Potapova, E., Zherebtsov, E., Kozlov, I., Seryogina, E., Kandurova, K., Alekseyev, A., Piavchenko, G., Kuznetsov, S., Mamoshin, A. and Dunaev, A., "Optical fine-needle aspiration biopsy in a rat model,” Proc. SPIE 10877, 108770K (2019). 